\title{
Gastrointestinal diseases among relatives of patients with esophageal atresia with or without tracheoesophageal fistula
}

\author{
Florian Zeck $^{1}$, Heiko Reutter ${ }^{1,2}$ \\ ${ }^{1}$ Department of Neonatology and Pediatric Intensive Care, Children's Hospital, University of Bonn, Bonn, Germany; ${ }^{2}$ Institute of Human Genetics, \\ University of Bonn, Bonn, Germany \\ Contributions: (I) Study conception and design: H Reutter; (II) Administrative support: H Reutter; (III) Provision of study materials or patients: H \\ Reutter; (IV) Collection and assembly of data: F Zeck; (V) Data analysis and interpretation: F Zeck; (VI) Manuscript writing: All authors; (VII) Final \\ approval of manuscript: All authors. \\ Correspondence to: Heiko Reutter. Department of Neonatology and Pediatric Intensive Care, Children's Hospital and Institute of Human Genetics, \\ University of Bonn, Sigmund-Freud-Str. 25, D-53127 Bonn, Germany. Email: reutter@uni-bonn.de.
}

\begin{abstract}
Background: Several studies have identified genetic factors that are associated with the formation of isolated and non-isolated esophageal atresia with or without tracheoesophageal fistula (EA/TEF) in human and mice. Some of these genetic factors like FOXF1/Foxf1 are associated with Barrett syndrome, esophageal carcinoma or tumors of the gastrointestinal tract. Here, we investigated the prevalence of common gastrointestinal diseases among EA/TEF patients and their first- and second-degree relatives (parents and grandparents).
\end{abstract}

Methods: We send out a questionnaire to $280 \mathrm{EA} / \mathrm{TEF}$ families asking for the presence of Barrett syndrome, Achalasia and carcinoma of the esophagus, the stomach, the small and large intestine among firstand second-degree relatives.

Results: In 32 of 124 families we found at least one affected family member with a possible association of colon carcinoma and the occurrence of EA/TEF within the same family.

Conclusions: Further studies are needed to evaluate a possible association.

Keywords: Esophageal atresia (EA); gastrointestinal disease; genetic risk factors

Submitted Jan 30, 2019. Accepted for publication Mar 29, 2019.

doi: $10.21037 /$ tp.2019.04.01

View this article at: http://dx.doi.org/10.21037/tp.2019.04.01

\section{Introduction}

Esophageal atresia with or without tracheoesophageal fistula (EA/TEF) represents the most common congenital anomaly of the upper digestive tract. The overall birth prevalence of EA/TEF ranges between 1 in 2,500 to 4,500 $(1,2)$. EA/TEF can be classified into five (Gross 1953) (3) respectively six (Vogt 1929) (4) subtypes depending on the classification system. Referring to Spitz (1) the most common subtype with $86 \%$ is type C according to Gross [1953], or type IIIb according to Vogt [1929], in which the proximal esophagus ends blindly in the superior mediastinum, whereas the distal esophagus enters the posterior wall of the trachea. In $45 \% \mathrm{EA} / \mathrm{TEF}$ presents as an isolated anomaly. In the remaining EA/TEF is associated with additional congenital anomalies as part of the VATER/ VACTERL association or within a defined monogenic syndrome such as Feingold syndrome, CHARGE syndrome and Fanconi anemia (5-7). Several murine knockout models presenting with EA/TEF as part of their phenotypic spectrum. Overall, these observations suggest that the causes of EA/TEF are highly heterogeneous (7). Among the identified underlying genetic factors are forkhead transcription factors, such as FOXF1 and FOXA2 (8-11). Interestingly, FOXF1 and FOXA2 have also been implicated in the formation of Barrett syndrome or esophageal adenocarcinoma respectively (8-12). Furthermore, EA/ 
TEF can be part of the Fanconi anemia complex, a genetic condition associated with an increased risk of developing acute myeloid leukemia (AML) or tumors of the head, neck, skin, gastrointestinal system, or genital tract. In this context a recent study by Panneerselvam et al. [2013] suggests a novel role of an inactivated FANCD2 in the upregulation of the oncogenic isoform of the p63 protein, delta Np63 $(\Delta \mathrm{Np} 63)$, supporting the idea that an impaired Fanconi anemia pathway contributes to the pathogenesis of certain human malignancies (12). As these genes participate in early embryonic foregut development and formation of EA/TEF and as these genes are also associated with gastrointestinal carcinomas and cancer precursors we thought to investigate the prevalence of common gastrointestinal carcinomas among EA/TEF patients and their first- and second-degree relatives (parents and grandparents). Furthermore, we also thought to investigate the prevalence of Barrett-syndrome and achalasia among EA/TEF patients and the first- and second-degree relatives.

\section{Methods}

\section{EA/TEF patients and families}

The study fulfilled with the requirement of the Declaration of Helsinki, and ethical approval was obtained from the local ethic committee (Lfd. Nr. 073/12 \& 146/12). Every participating family provided written informed consent. Families were re-contacted within the context of two German multicenter studies on the genetic and environmental causes of: (I) EA/TEF ["The genetic risk for esophageal atresia-consortium (great-consortium)"]; and (II) ARM ["Network for the Systematic Investigation of the Molecular Causes, Clinical Implications, and Psychosocial Outcome of Congenital Uro-Rectal Malformations (CURE-Net)]".

\section{Gastrointestinal cancer: German Centre for Cancer Registry Data of the Robert Koch Institute}

The German Centre for Cancer Registry Data of the Robert Koch Institute registers incidents and prevalence of every cancer in Germany. The latest data available to compare the prevalence of esophageal cancer, stomach cancer and bowel cancer is from 2014. The data shows the crude rate of the 5 -year prevalence for each cancer subdivided by the sex and different age groups. Small intestine cancer and colon cancer are not registered individually.

\section{Questionnaires}

We send out 280 questionnaires (families of greatconsortium $n=230$; families of CURE-Net $n=50$ ). In order to include as many families as possible, we additionally recontacted all families by phone. The questionnaire asked for the presence of Barrett syndrome, achalasia, carcinoma of esophagus, stomach, small intestine, and colon. We asked for the occurrence of these diseases among parents and grandparents of the respective patient. The questionnaire did not include at what age the disease occurred and at what age the respective family members had died if they were dead. All patients were born between 1970 and 2015. Thirteen patients had VATER/VACTERL association, two had Goldenhar syndrome.

\section{Results}

\section{Questionnaire return rate}

Of the 280 questionnaires sent out, the return rate was $44 \%$ ( $\mathrm{n}=124$ families). These 124 families comprised 248 firstdegree relatives and 496 second-degree relatives. Fifty-nine of EA/TEF patients were aged $0-15$ years of age; $53 \mathrm{EA} /$ TEF patients were 15-30 years of age, and $12 \mathrm{EA} / \mathrm{TEF}$ patients were $>30$ years of age.

\section{First- and second-degree relatives (parents and grandparents)}

In 92 families none of the first- or second-degree relatives had Barrett syndrome, achalasia, carcinoma of esophagus, stomach, small intestine or colon at the time of assessment. In $32(\mathrm{P}=0.26)$ families there was at least one family member who was affected by Barrett syndrome, achalasia, carcinoma of esophagus, stomach, small intestine or colon at the time of assessment.

In 22 families one family member was affected, in nine families two members were affected and in one family three family members were affected by one or two diseases. The information on two mothers and on four fathers, 12 grandmothers and 13 grandfathers remained incomplete even after personal inquiry.

\section{Barrett syndrome}

The Barrett syndrome occurred three times. Two of these three affected with Barrett syndrome came from the same family and concerned the mother and the maternal grandfather of the EA/TEF affected patient. As there is no 
Table 1 Prevalence of investigated gastrointestinal disease in firstand second-degree relatives

\begin{tabular}{|c|c|c|c|c|}
\hline \multirow{2}{*}{ Disease } & \multicolumn{2}{|c|}{ First-degree relatives } & \multicolumn{2}{|c|}{ Second-degree relatives } \\
\hline & Mother & Father & Grandmother & Grandfather \\
\hline $\begin{array}{l}\text { Barrett } \\
\text { syndrome }\end{array}$ & 1 & 0 & 1 & 1 \\
\hline Achalasia & 0 & 0 & 0 & 1 \\
\hline $\begin{array}{l}\text { Esophageal } \\
\text { CA }\end{array}$ & 0 & 0 & 2 & 4 \\
\hline Stomach CA & 0 & 0 & 2 & 8 \\
\hline $\begin{array}{l}\text { Small } \\
\text { intestine CA }\end{array}$ & 1 & 0 & 3 & 1 \\
\hline Colon CA & 1 & 2 & 9 & 12 \\
\hline
\end{tabular}

CA, carcinoma.

population to compare it to, we cannot tell if the prevalence of Barrett syndrome among first- and second-degree relatives of EA/TE patients is elevated.

\section{Achalasia}

Achalasia appeared once in combination with colon carcinoma in a second-degree relative. Birgisson (13) describes the prevalence of Achalasia in Iceland with 8.7/100,000 and Sadowski et al. (14) describes the prevalence with $10.8 / 100,000$. As in our study only one second-degree relative presented with Achalasia at the time of assessment, we did not perform statistical analysis on a possible association here.

\section{Gastrointestinal carcinoma}

Esophageal carcinoma occurred six times in seconddegree relatives. In one instance carcinoma of the colon co-occurred with carcinoma of the esophagus. In one firstand in four second-degree relatives carcinoma of the small intestine occurred of which three were combined with carcinoma of the colon. In three first-degree relatives and in 21 second-degree relatives carcinoma of the colon occurred (Table 1). Our questionnaire did not differentiate between polypose and non-polypose colon cancer.

A comparison of the results to the data of the German Centre for Cancer Registry Data is not possible, as the information on age of the second-degree relatives is incomplete and the number of cases of the first-degree relatives is too small to compare it (Table 2). To show the problem in an example: with a crude rate of 135 affected in
100,000 males between 45-54 years of age we would have had to interrogate at least 740 men of that age group to find out if the prevalence had been elevated or not elevated.

Of all first- and second-degree relatives that participated $(n=713), 3.4 \%$ had colon carcinoma. These relatives range with their age between 37 and 82 . When looking at population based data the life risk of colon carcinoma in Germany for the age range $0-84$ is between $3.7-6.4 \%$ (15). Hence, in this explorative comparison, our data suggest that we need further analysis from large EA/TEF study groups in order to re-evaluate a possible association of colon carcinoma and the occurrence of EA/TEF within the same family.

\section{Discussion}

Previous studies showed that several genes involved in the formation of mainly non-isolated/syndromic EA/ TEF were also involved in the pathogenesis of human gastrointestinal carcinoma (12). The present study investigated the prevalence of Barrett syndrome, Achalasia and gastrointestinal carcinomas among first- and seconddegree relatives (parents and grandparents) of EA/TEF patients. To the best of our knowledge, this is the first study to investigate such associations among EA/TEF families. Of the 280 questionnaires sent out, the return rate was $44 \%$ ( $\mathrm{n}=124$ families). It is unclear, why the return rate was below $50 \%$, as our questionnaire was purely explorative and restricted to six questions each family member only. Furthermore all families addressed had previously consented to participate in our genetic and epidemiologic study on the causes of EA/TEF.

When looking at first- and second-degree relatives of EA/TEF patients we found in 32 of 124 families at least one affected family member with Barrett syndrome, Achalasia and/or gastrointestinal carcinoma.

Most of these affected first- and second-degree relatives had colon carcinoma $(n=24)$. While our questionnaire did not differentiate between polypose and non-polypose colon cancer, we did not find any previously described association between congenital anomalies of the esophagus and any form of colon carcinoma in the literature. An explorative comparison between the here observed prevalence of colon carcinoma and population-based data suggest that we need to re-evaluate a possible association of colon carcinoma and the occurrence of EA/TEF within the same family within the context of large EA/TEF study groups.

Our preliminary analysis on the co-occurrence or association of Barrett syndrome, achalasia or gastrointestinal 
Table 2 Prevalence in first-degree relatives of carcinoma of the small intestine and the colon in comparison to the data of the German Centre for Cancer Registry Data

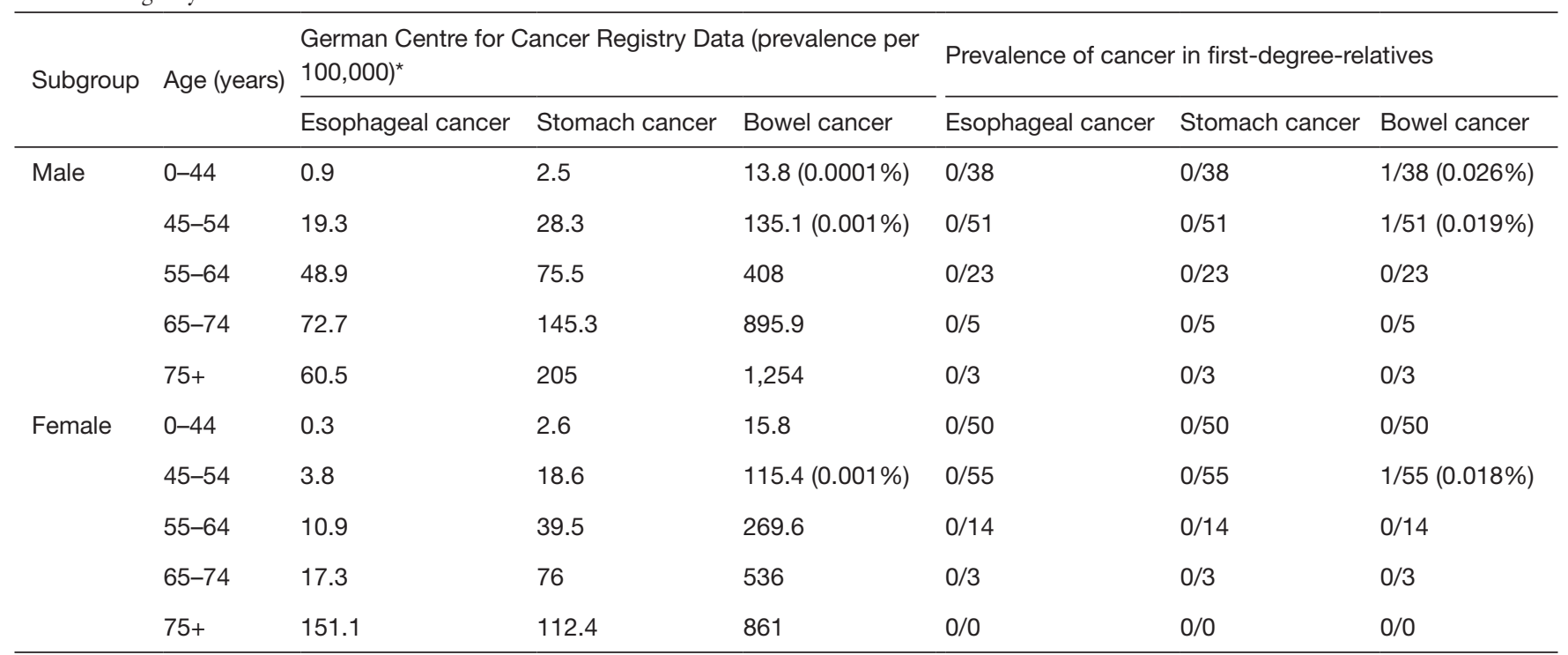

*, German Centre for Cancer Registry Data: crude rate of 5-year prevalence in Germany per 100,000 people [2014].

carcinomas among EA/TEF patients has several major limitations. First, the assessment was made through a patient/family based self-assessment questionnaire. While all families who reported a positive history where recontacted personally by the author FZ to confirm the diagnosis of the affected family members, we did not assess how thorough all family members were evaluated for the presence of Barrett syndrome, achalasia or gastrointestinal carcinomas. Furthermore, family members who deceased in the past with unknown cause were not further investigated. Third, the EA/TEF cohort investigated was relatively young, rendering their risk for developing Barrett syndrome, achalasia or gastrointestinal tumors relatively low. Finally, or study cohort is not population based but has been re-contacted through two German wide studies on the "The genetic risk for esophageal atresiaconsortium (great-consortium)" and the "Network for the Systematic Investigation of the Molecular Causes, Clinical Implications, and Psychosocial Outcome of Congenital Uro-Rectal Malformations (CURE-Net)".

\section{Conclusions}

Our study suggests that the occurrence of common gastrointestinal disease among first- and second-degree relatives of EA/TEF patients needs to be re-evaluated in large EA/TEF study groups. Furthermore we suggest a possible association of colon carcinoma and the occurrence of EA/TEF within the same family.

\section{Acknowledgments}

We thank all patients and their families for their participation, as well as the German self-help organizations for individuals with anorectal malformations (SoMA e.V.) and tracheoesophageal fistula with or without esophageal atresia (TE) (KEKS e.V.) for their assistance with recruitment.

\section{Footnote}

Conflicts of Interest: The authors have no conflicts of interest to declare.

Ethical Statement: The study fulfilled with the requirement of the Declaration of Helsinki, and ethical approval was obtained from the local ethic committee (Lfd. Nr. 073/12 \& 146/12). Every participating family provided written informed consent.

\section{References}

1. Spitz L. Oesophageal atresia. Orphanet J Rare Dis 2007;2:24. 
2. Nassar N, Leoncini E, Amar E, et al. Prevalence of esophageal atresia among 18 international birth defects surveillance programs. Birth Defects Res A Clin Mol Teratol 2012;94:893-9.

3. Gross RE. The Surgery of infancy and childhood. Philadelphia, PA: WB Saunders, 1953.

4. Vogt EC. Congenital esophageal atresia. AJR Am J Roentgenol 1929;22:463-5.

5. Chittmittrapap S, Spitz L, Kiely EM, et al. Oesophageal atresia and associated anomalies. Arch Dis Child 1989;64:364-8.

6. Shaw-Smith C. Oesophageal atresia, tracheo-oesophageal fistula, and the VACTERL association: review of genetics and epidemiology. J Med Genet 2006;43:545-54.

7. Bednarczyk D, Sasiadek MM, Smigiel R. Chromosome aberrations and gene mutations in patients with esophageal atresia. J Pediatr Gastroenterol Nutr 2013;57:688-93.

8. Wang DH, Tiwari A, Kim ME, et al. Hedgehog signaling regulates FOXA2 in esophageal embryogenesis and Barrett's metaplasia. J Clin Invest 2014;124:3767-80.

9. Mahlapuu M, Enerbäck S, Carlsson P. Haploinsufficiency of the forkhead gene Foxf1, a target for sonic hedgehog signaling, causes lung and foregut malformations. Development 2001;128:2397-406.

10. Merchant JL. Hedgehog signalling in gut development, physiology and cancer. J Physiol 2012;590:421-32.

11. Becker J, May A, Gerges C, et al. Supportive evidence for FOXP1, BARX1, and FOXF1 as genetic risk loci for the development of esophageal adenocarcinoma. Cancer Med 2015;4:1700-4.

12. Panneerselvam J, Pickering A, Zhang J, et al. A hidden role of the inactivated FANCD2: upregulating $\triangle \mathrm{Np} 63$. Oncotarget 2013;4:1416-26.

13. Birgisson S, Richter JE. Achalasia in Iceland, 1952-2002: an epidemiologic study. Dig Dis Sci 2007;52:1855-60.

14. Sadowski DC, Ackah F, Jiang B, et al. Achalasia: incidence, prevalence and survival. A population-based study. Neurogastroenterol Motil 2010;22:e256-61.

15. Brenner H, Schrotz-King P, Holleczek B, et al. Declining Bowel Cancer Incidence and Mortality in Germany. Dtsch Arztebl Int 2016;113:101-6.
Cite this article as: Zeck F, Reutter H. Gastrointestinal diseases among relatives of patients with esophageal atresia with or without tracheoesophageal fistula. Transl Pediatr 2019;8(5):378-382. doi: 10.21037/tp.2019.04.01 